A N N A L E S Annales de Bretagne et des Pays de l'Ouest

Anjou. Maine. Poitou-Charente. Touraine

111-1 | 2004

Varia

\title{
Le Front populaire et les conserveurse sardines de Bretagne
}

Jean-Christophe Fichou

\section{OpenEdition}

Édition électronique

URL : http://journals.openedition.org/abpo/1307

DOI : $10.4000 /$ abpo. 1307

ISBN : 978-2-7535-1493-5

ISSN : 2108-6443

Éditeur

Presses universitaires de Rennes

Édition imprimée

Date de publication : 20 mars 2004

Pagination : 111-125

ISBN : 978-2-86847-976-1

ISSN : 0399-0826

\section{Référence électronique}

Jean-Christophe Fichou, «Le Front populaire et les conserveurse sardines de Bretagne », Annales de Bretagne et des Pays de l'Ouest [En ligne], 111-1 | 2004, mis en ligne le 20 mars 2006, consulté le 02 mai 2019. URL : http://journals.openedition.org/abpo/1307 ; DOI : 10.4000/abpo.1307 


\title{
Le Front populaire et les conserveurs de sardines de Bretagne ${ }^{1}$
}

\author{
Jean-Christophe FICHOU \\ Professeur d'histoire et géographie \\ collège de Kerichen, Brest
}

Au printemps 1936, 150 conserveries de sardines à l'huile fonctionnent sur l'ensemble du littoral atlantique entre Camaret et Saint-Jean-de-Luz. À leur tête on dénombre une centaine de conserveurs dont les intérêts et les combats politiques ou économiques sont le plus souvent similaires. Ils viennent de subir de plein fouet les effets de la crise de 1929, et se relèvent difficilement de cette épreuve; l'annonce de la victoire de l'union des gauches en 1936 est pour eux une effroyable nouvelle.

\section{Les réactions patronales au gouvernement de Front populaire}

En janvier 1936 est publié le programme du Front Populaire qui est né quelques mois auparavant, en juillet 1935. La SFIO, les radicaux et le Parti Communiste, unis pour la circonstance, obtiennent la majorité absolue en sièges aux élections législatives des 26 avril et 3 mai 1936. Ce succès réveille chez les ouvriers un grand espoir marqué par une première vague de grèves sans précédent; elles commencent au Havre le 11 mai et s'achèvent le 11 juin après la signature des accords Matignon par la CGT et la CGPF. En Bretagne, les 10700 ouvriers et ouvrières ${ }^{2}$ employés des 149 conserveries de poisson ouvertes $^{3}$ ce printemps-là et qui jalonnent le littoral du Finistère, du Morbihan et de la Loire-Inférieure, suivent certainement avec grand intérêt l'évolution de la situation mais ne se manifestent pas, du moins publiquement. Pourtant, la nouvelle de cette victoire est sans doute mal acceptée en Bretagne où les électeurs se sont prononcés en majorité pour les candidats hostiles au Font populaire et ont permis l'élection de trente députés, sur quarante-quatre, de droite et centre-droite ${ }^{4}$ opposés au gouvernement Blum.

1. Je tiens à remercier Christian Bougeard pour ses suggestions et ses corrections.

2. Statistiques des Pêches maritimes pour l'année 1936, Paris, Imprimerie nationale, 1938.

3. Archives Syndicat des conserveurs, Quimper. Rapport d'activité, 1936.

4. BougEard, Christian, "Les notables et les forces politiques de droite en Bretagne dans les années 1930 ", Annales de Bretagne et des Pays de l'Ouest, tome 109/3, 2002, p. 121. 
"On saurait difficilement exagérer l'émoi que, dans les rangs des classes aisées, même parmi les hommes en apparence les plus libres d'esprit, provoqua, en 1936, l'avènement du Front populaire... Une longue fente, séparant en deux blocs les groupes sociaux, se trouva, du jour au lendemain, tracée dans l'épaisseur de la société française ${ }^{5}$."

Les représentants du patronat ont signé dans l'effroi, la précipitation et l'ignorance mais l'ensemble des dirigeants d'entreprise, la surprise passée, réagissent rapidement, fébriles et inquiets cependant. Les conserveurs notamment, comme les autres entrepreneurs, s'empressent de vider leurs comptes. À Quimper, les banques reçoivent, au mois de mai, des ordres de vente de valeurs d'État plus nombreux que d'habitude. Il en est de même pour les demandes de valeurs étrangères effectuées par des clients importants. La préfecture du Finistère est rapidement au courant et demande des informations complémentaires :

« Il y aurait lieu de procéder [à des] enquêtes semblables à Douarnenez, à Concarneau et à Pont-l'Abbé où des opérations pourraient avoir été faites pour le compte de gros industriels de la conserve ${ }^{6}$."

Chez les conserveurs bretons, ni les syndicats patronaux ni les Chambres de Commerce de Brest, de Quimper, de Lorient et de Nantes ne s'expriment dans l'instant, sans doute parce qu'ils ne sont pas informés très précisément de la nature exacte des accords et surtout de leur portée. Si aucune allusion n'est faite au sujet des acquis sociaux obtenus par les ouvriers dans les procès verbaux des séances, en mai et juin 1936, il est clair que les patrons en général et les conserveurs en particulier n'acceptent que difficilement cette situation humiliante qui leur échappe en grande partie. Ils subissent " l'impact d'un retard historique, d'un manque de perspicacité, de lucidité et de prévoyance ${ }^{7}$ ", mais s'en rendent-ils vraiment compte? Pourtant la période de flottement, si elle a existé, a été de courte durée; la réaction, celle des assemblées consulaires en particulier, ne se fait pas attendre. Pierre-Ernest Dalbouze, le président de la Chambre de Commerce de Paris, convoque dès le 9 juin 1936 les présidents des 114 Chambres françaises et obtient l'accord de ces derniers sur un texte très critique à l'égard du gouvernement :

"L'Assemblée des présidents des Chambres de Commerce réunie en session extraordinaire [...] constate que le principe de la propriété privée et celui de la liberté individuelle ont été violés ouvertement; [...] rappelle que la confiance, condition primordiale du redressement économique, commande le maintien absolu de l'ordre public; [...] a le devoir, dans l'intérêt même des travailleurs, de mettre en lumière les risques et les conséquences de mesures incompatibles avec une situation dont les employeurs ne sont pas plus maîtres que leur personnel; $[\ldots]$ attire l'attention des Pouvoirs

5. Bloch, Marc, L'Étrange défaite, Paris, Gallimard, 1946, [rééd. 1990], p. 197.

6. Arch. dép. du Finistère, 1 M 188, Surveillance de l'opinion. Quimper, le 12 mai 1936, le commissaire au préfet.

7. KolboOm, Ingo, La Revanche des patrons, le patronat français face au Front populaire, Paris, Flammarion, 1986, p. 36. 
publics sur la gravité de la responsabilité qui leur incomberait en imposant à l'économie française $[\ldots]$ une charge intolérable génératrice d'un bouleversement total de la position économique du Pays ${ }^{8}$."

Dalbouze est secondé dans son entreprise de mobilisation patronale par son vice-président, Pierre Lemy, par ailleurs président du Syndicat des Fabricants de conserves de Paris et propriétaire d'usines aux Sables d'Olonne, à Plomeur et à Lesconil ${ }^{9}$. Un mois et demi plus tard, le 20 juillet 1936, Dalbouze obtient de nouveau les signatures de tous les représentants; sa seconde résolution prévoit que le gouvernement Blum ne puisse appliquer les mesures sociales sans concertation préalable avec tous les acteurs de la vie économique ${ }^{10}$ et pas seulement les représentants des très grandes entreprises. La CGPF n'est pas en reste : accusée de mollesse, voire de trahison par un petit et moyen patronat qui ne se reconnaît pas dans les concessions de Matignon, elle doit reprendre les choses en main. Après le 4 août elle représente désormais le Patronat français et non plus la Production française. Son nouveau directeur, Claude-Joseph Gignoux, agrégé de droit, est nommé pour retrouver et restaurer l'autorité patronale pleine et entière dans l'entreprise ${ }^{11}$. Très vite aussi, les fabricants de conserves bretons et vendéens, par l'entremise de leurs syndicats régionaux et de l'Union nationale, réagissent et présentent leurs doléances au gouvernement; elles sont portées par Pierre Lemy. Il s'agit des mêmes plaintes que celles présentées par l'ensemble du patronat : les augmentations de salaires de 7 à $15 \%$ sont difficilement acceptables pour les petites entreprises, surtout les conserveries, industrie de main d'œuvre par excellence. La semaine des quarante heures est massivement rejetée, les conventions collectives et la reconnaissance du fait syndical sont très mal perçues par les usiniers qui n'entendent pas perdre la plénitude de leur autorité. Très vite, les opinions se radicalisent et la présence de Schang, conserveur et président du Syndicat des Conserveurs bretons, et celle de son collègue, Marcel Chacun, membre de la Chambre de Commerce de Quimper, à la réunion organisée par le PSF à Quimper le 10 octobre 1936, est particulièrement remarquée ${ }^{12}$. Édouard Paulet est aussi un militant actif de ce parti

8. Archives CCI Brest, Archives CCI Quimper; lecture en séance à Brest et Quimper des résolutions votées par leurs présidents respectifs le 9 juin 1936 lors de l'assemblée extraordinaire à Paris.

9. Ils peuvent aussi compter sur l'actif concours du trésorier de l'Assemblée des Chambres de Commerce de France et d'Algérie, Francis Merlant, conserveur, arrière-petit fils de Maurice Amieux, marié à une nièce de Louis Amieux et membre de la Chambre de Commerce de Nantes.

10. Archives CCI Lorient, séance d'août 1936.

11. RichaRD, Gilles, "Claude-Joseph Gignoux : une biographie exemplaire? ", Annales de Bretagne et des Pays de l'Ouest, tome 109, 2002, p. 141-155. Est citée, page 143, l'opinion de Blum qui ne l'appréciait pas : "Un de ces hommes qui sont étrangers au patronat et qui, soit par esprit de parti, soit par fanatisme doctrinal, apportent parfois à la défense des intérêts patronaux plus d'âpreté que les patrons eux-mêmes. "

12. Arch. dép. du Finistère, 1 M 229, partis royalistes et Action française. Quimper le 11 octobre 1936, le commissaire. 
et il organise de nombreuses rencontres pour développer les idées du lieutenant colonel de La Roques ${ }^{13}$. Personne ne peut ignorer l'engagement de Jacques Guillemot, gendre de Pierre Chancerelle et directeur de l'usine Chancerelle de Quimper, ancien militant de la ligue dissoute de l'Action française ${ }^{14}$ surpris à placarder des affiches condamnant la République et le parlementarisme, "Règne de l'irresponsabilité, l'instabilité, l'incompétence, l'injustice ${ }^{15}$... ". Ce même Guillemot organise, le 22 novembre 1937 à Quimper, une réunion privée, sous les auspices de l'Action Nationale et Sociale (ANS), au cours de laquelle Héricourt, journaliste au Charivari, prononce un discours intitulé : "Quelques semaines sur le front espagnol, reportage vécu sur les atrocités marxistes ". Il est facile de conclure, comme le commissaire spécial, que l'ANS n'est que la " continuation à peine déguisée de la Ligue d'Action française ${ }^{16}$ ".

En Bretagne, les grèves ne touchent que peu, ou de manière diffuse, le secteur de la conserve au cours de la première période, lors de la grande vague de mai-juin. Par contre les mouvements se multiplient au début de l'été avec le commencement de la campagne sardinière, et pour répliquer aux réticences des usiniers peu enclins à répondre aux termes des accords de Matignon ${ }^{17}$. Indirectement aussi la conserverie bretonne est frappée; les Forges sont touchées en mai et juin par les grèves ${ }^{18}$. Les fabricants de boîtes et les usiniers s'inquiètent car les livraisons de fer-blanc se font attendre. Ainsi la Société Métallurgique de Douarnenez demande à la préfecture d'intervenir pour hâter la venue du chargement tant convoité :

"Nous avons des marchés réguliers avec différentes forges. Celles-ci par suite des conflits qui se sont terminés nous disent être désorganisées et invoquent le cas de force majeure pour retarder les livraisons ${ }^{19}$."

\section{Le refus du " diktat " de Matignon}

Bien entendu, l'application des accords Matignon ne s'effectue pas dans le calme souhaité par le gouvernement : l'Union nationale et le Syndicat breton des conserveurs s'opposent farouchement aux nouvelles dispositions

13. Entretiens Dominique Paulet. Par ailleurs il faut savoir que Pierre Édouard Paulet s'embarque par la Grande-Bretagne dès le 18 juin 1940. Âgé de 43 ans, il s'engage dans les forces françaises libres et meurt en août 1942 en Méditerranée.

14. Arch. dép. du Finistère, 1 M 227, Partis de droite. Décret du 18 juin 1936 portant dissolution de l'association déclarée dite le Mouvement Social Français des Croix de Feu et de toutes les ligues; le 21 juin est fondé le PSF puis le 28 juin le PPF.

15. Arch. dép. du Finistère, 1 M 229. Quimper, août 1936, le commissaire.

16. Arch. dép. du Finistère, 1 M 188, surveillance de l'opinion. Quimper, le 25 janvier 1937, le commissaire au préfet.

17. BOUGEARD, Christian, La Bretagne d'une guerre à l'autre, 1914-1945, Paris, Gisserot, 1999, p. 61.

18. Forges de Wendel, platinerie de Hayange; Forges de Basse-Indre; Forges d'Hennebont; Forges de la Gironde...

19. Arch. dép. du Finistère, 4 S 364, Douarnenez, le 20 juillet 1936, la Société Métallurgiste de Douarnenez au préfet. 
de la loi, et surtout aux quarante heures hebdomadaires de travail, texte voté malgré les protestations énergiques des fabricants. Les ouvriers réagissent aussitôt : les grèves des conserveries de Penmarc'h (12 juin), de Camaret (du 14 au 18 septembre), de Concarneau (du premier au 19 septembre) le prouvent sans contestation possible, les salariés réclament tous l'application des accords ${ }^{20}$. Et la manière forte est payante : les filles de chez Provost-Barbe, à Concarneau, sans grève ni cessation de travail, réclament à leur patron les augmentations prévues par la loi : elles obtiennent trente centimes de plus par heure et quatre-vingts centimes après dix heures de travail consécutives. Les ouvriers de l'usine Chatelard de Concarneau décident la grève le 12 juin, sans tenir compte du désormais célèbre apophtegme de Maurice Thorez, " il faut savoir terminer une grève ${ }^{21}$ ", prononcé le jour précédent, pour obtenir une augmentation uniforme de cinquante centimes de l'heure et, ce qui est une première dans le monde des usines de conserves, divers aménagements relatifs à l'hygiène intérieure. De son côté le directeur promet 10 \% d'augmentation sur les salaires mensuels. Un accord intervient à 20 heures : les femmes ont obtenu 1,80 franc de l'heure, les manœuvres trois francs et les ajusteurs quatre francs, ce qui reste encore dérisoire par rapport aux moyennes ouvrières nationales. Dans tous les cas, il s'agit de mouvements quasi spontanés, courts, jamais préparés de longue date et encore moins encadrés par des militants de la CGT. Au début de l'été, les conserveurs répondent tous favorablement aux demandes de leurs ouvriers. Le commissaire de police de Concarneau constate que les usiniers se plient désormais aux recommandations gouvernementales sans renâcler ouvertement et sans incident majeur :

" En application de l'accord de Matignon conclu à Paris le 7 juin 1936 entre la CGPF et la CGT sous l'arbitrage de M. Léon Blum, Président du Conseil, et signé par lui, les salaires sont augmentés à partir du 10 juin 1936, chacun dans la proportion de ce contrat ${ }^{22}$."

À Douarnenez, les ouvriers ne sont guère combattifs car les usiniers, ici aussi, accordent les augmentations réclamées. La municipalité de Daniel le Flanchec n'a guère de problèmes à régler durant cette période ${ }^{23}$; tout juste vote-elle un crédit de 1000 francs pour acheter des conserves de poissons dans une usine de la ville " pour être expédiées au Gouvernement du Front populaire d'Espagne ${ }^{24}$ ". Pourtant les grèves reprennent peu à peu dans les petits ports alentours, où les usines et la population ouvrière sont

20. Arch. dép. du Finistère, $10 \mathrm{M}$ 64, grèves 1936.

21. "Alors il faut savoir terminer une grève dès que satisfaction a été obtenue. Il faut même savoir consentir au compromis si toutes les revendications n'ont pas encore été acceptées, mais si l'on a obtenu la victoire sur la plus essentielle des revendications. Tout n'est pas possible. " Cité dans KeRGOAT, Jacques, La France du Front populaire, Paris, La Découverte, 1986, p. 124.

22. Arch. dép. du Finistère, $10 \mathrm{M}$ 64, Concarneau, le 13 juin 1936, le commissaire au préfet. 23. Le Boulanger, J.-M., Douarnenez de 1800 à nos jours, Rennes, PUR, 2000, p. 237.

24. Arch. dép. du Finistère, 1 M 188, surveillance de l'opinion; Douarnenez, délibération du 16 septembre 1936. 
moins nombreuses, car les conserveurs violent ou tournent à " qui mieux mieux les nouvelles lois sociales ${ }^{25}$ ". Les ouvrières de l'usine Cassegrain de Saint-Guénolé, lassées d'attendre les augmentations promises, cessent le travail le 21 août et réclament leur dû. Cependant, après quatre jours de grève, elles reprennent le chemin de l'usine dans les mêmes conditions qu'auparavant sans avoir obtenu satisfaction. Ces arrêts de travail sans cesse renaissants mais décidés en ordre dispersé n'ont guère de chance d'aboutir et les combats sont facilement repoussés par les usiniers. Ils ont repris de l'assurance, ils contre-attaquent et interviennent bruyamment pour souligner les particularités de leur profession : le caractère saisonnier de la pêche, le travail aléatoire et toujours fonction des apports, la nécessité de travailler vite toutes les sardines débarquées sans tenir compte des horaires, et les salaires qu'on ne peut augmenter sans mettre en danger toute la profession... Pour eux les décisions de Matignon ne peuvent être respectées et ils profitent de la " pause " annoncée par Blum en février 1937 pour faire valoir leur opinion.

"C'est là une augmentation impossible à concevoir aussi est-il à craindre que les usiniers ne restreignent volontairement leur production pour échapper aux salaires prohibitifs qu'ils seront contraints de payer si l'application du décret se fait d'une manière intransigeante ${ }^{26}$."

Rien n'y fait pourtant, toutes leurs observations, plaintes ou reproches restent vains, et le régime de faveur auquel les gouvernements successifs les avaient habitués n'est toujours pas accordé. Dès lors, le ton se durcit et les propos des conserveurs deviennent beaucoup plus acerbes :

"Nous nous sommes trouvés en face de doctrinaires bornés qui ne veulent pas comprendre que le soleil et la pluie agissent en dehors de toute volonté $^{27}$."

Et si les rapports insultants ne réussissent pas à convaincre le gouvernement, on reprend le concert des lamentations comme le fait Queinnec, membre de la Chambre de Commerce de Brest et conserveur au Faou, dans un discours intitulé "L'Agonie de la petite et moyenne industrie " :

"Le vote récent des lois sociales au profit de la classe ouvrière à des répercussions malheureuses sur les trésoreries appauvries des petites industries par ces dernières années de crise. On a attendu qu'elles soient à l'agonie pour s'en occuper... Si certains se réjouissent de cette situation qui facilitera la disparition d'un élément ordonné de la Nation, n'oublions pas qu'elle contribuera à la nationalisation des grosses firmes qui subsisteront. Est-ce cela que l'on cherche ${ }^{28}$ ?"

Un an après la signature des accords de Matignon, l'attitude des conserveurs est encore plus stricte, brusque, voire injurieuse; ils n'oublient pas

25. GuERIN, Daniel, Front populaire. Révolution manquée, Paris, Actes Sud, 1970, [rééd. 1997], p. 219.

26. Archives CCI Quimper, Quimper, le 18 avril 1937, le président de Servigny.

27. Archives Syndicats des conserveurs, Quimper, le 7 avril 1938.

28. Archive CCI Brest, discours lu en séance le 15 avril 1937. 
de rappeler violemment lors du premier anniversaire de l'installation du gouvernement Blum :

" [...] le préjudice certain que la mise en application de la loi des 40 heures apporte aux marins-pêcheurs. Il faut que les pouvoirs publics portent la responsabilité de leur impéritie, de leur entêtement à ne pas vouloir tenir aucun compte des observations que le Syndicat [des conserveurs] a faites et qui sont restées lettres mortes ${ }^{29}$."

Démesure des usiniers au lendemain d'une bataille éprouvante? Certainement, mais les propos sont imprimés, publiés et distribués par la Chambre de Commerce de Quimper une fois le calme revenu. Une telle attaque contre le président du Conseil et ses ministres révèle l'état d'esprit partagé par l'ensemble de la profession. Très vite cependant, ils se rendent compte qu'il est trop difficile de refuser de front la loi; ils tentent alors d'imposer leur point de vue, d'une part en laissant pourrir la situation, en accordant les augmentations à reculons, et d'autre part en utilisant à leur avantage les articles de loi. Le 7 juin 1937 la grève générale est décidée à Concarneau et, grande première dans le monde industriel sardinier, elle est déclenchée sur ordre de la CGT, afin de provoquer la décision surarbitrale le plus rapidement possible. Il faut se rappeler que la ville est alors dirigée depuis 1935 par Pierre Guéguin, maire communiste ${ }^{30}$, si bien que l'intervention de la CGT s'explique sans doute plus aisément. Les syndicalistes constatent que les accords Matignon ne sont pas respectés et dénoncent la mauvaise volonté des usiniers. Pour la première fois aussi dans l'histoire des conflits de la conserve, des piquets de grève sont placés devant les usines Rödel, Provost, Teysonneau, Pennanros, Cassegrain... Il est sans doute vrai que la grève aurait connu le même sort sans l'aval et l'organisation de la Confédération. Pour autant la réaction patronale est ferme et immédiate, d'autant plus que le mouvement est justement dirigé par la CGT :

"Cette grève a été déclenchée dans des conditions extrêmement illégales puisque le seul différend que nous connaissons actuellement et qui a trait à une demande adressée par les organisations ouvrières au Syndicat de Bretagne est soumise à la procédure de l'arbitrage conformément à la loi... En conséquence, nous envoyons à tous nos hommes une lettre recommandée avec accusé de réception les informant qu'ils ne font plus partie de notre personnel ${ }^{31}$."

Le jour même, tous les patrons concernés répondent immédiatement par le licenciement individuel de tous leurs ouvriers. Un accord intervient quand même le 10 juin et le travail reprend le 11 sans qu'aucune sanction

29. Archives CCI Quimper, le 26 mai 1937, le président du Syndicat des conserveurs à la Chambre de Commerce.

30. Pierre Guéguin, né le 18 août 1896 à Quimerc'h, professeur de l'école primaire supérieure, maire de Concarneau depuis 1935. Il est déchu de ses fonctions en 1940 car il refuse de renoncer à ses idées politiques communistes. Il est fusillé le 22 octobre 1941 au nombre des Cinquante otages de Chateaubriand.

31. Arch. dép. du Finistère, 10 M 64, Concarneau, le 7 juin 1937. Cette lettre reproduite à l'identique par neuf industriels de la conserve est adressée au préfet du Finistère. 
particulière ne soit appliquée, mais les salariés n'obtiennent pas les augmentations de salaire demandées. Les conserveurs appliquent à la lettre les consignes du président Dalbouze adressées à toutes les Chambres de Commerce de France quelques mois auparavant :

" Je tiens [...] à insister auprès de vous sur l'attitude nette et énergique qui s'impose en présence d'occupations, soit massives, soit effectuées sous forme de piquets de grève. J'attire personnellement votre attention [...] sur l'heureuse influence d'une attitude de fermeté ${ }^{32} \ldots$ ".

\section{Une lente " deffervescence ${ }^{33}$ "}

À n'en pas douter la magie du Front populaire s'est bien dissipée, d'autant plus que Blum doit abandonner le 21 juin 1937 la présidence du Conseil au profit de Camille Chautemps, un radical plus acceptable aux yeux des usiniers ${ }^{34}$. Les conserveurs profitent de cette passation de pouvoir et avancent une nouvelle argumentation en jouant sur les termes du temps de travail; en considérant que les quarante heures effectuées en cinquante semaines reviennent à travailler 2000 heures légales par an les fabricants parviennent à cette conclusion limpide :

«Il est décidé que nous voulons la libre disposition des 2000 heures par an avec si possible 500 heures de dérogation légale suivant les nécessités du travail. Notre industrie est en effet unique en son genre. L'application brutale de la loi ne peut donc nous être imposée ${ }^{35}$."

Comme les conserveries françaises ne travaillent que 120 à 150 jours par an, cela signifie concrètement un travail quotidien de 14 à 17 heures pour les ouvrières sans avantage financier : la situation n'évolue absolument pas. Le gouvernement reconnaît enfin le caractère saisonnier de l'activité et admet qu'il est impossible d'appliquer la loi des 40 heures dans cette branche industrielle. La CGT n'apprécie que modérément l'attitude de l'État et manifeste dès lors une opposition beaucoup plus marquée et plus systématique envers les usiniers. Les luttes revendicatives prennent un caractère politique de plus en plus prononcé ${ }^{36}$. Mais les effectifs féminins du syndicat demeurent relativement faibles; on note pourtant que de plus en plus d'ouvrières rallient les rangs de l'Union régionale reconnaissant l'attitude plus belliqueuse de la Confédération sans pour autant en adopter massivement la ligne. Bien sûr la CGT profite de l'occasion offerte et elle

32. Archives CCI Brest. Paris le 22 septembre 1936, le président Dalbouze au président Corre.

33. KeRGOAT, Jacques, La France du Front populaire, op. cit., p. 125.

34. Le 15 juin 1937 Léon Blum demande au Parlement les pleins pouvoirs financiers : la Chambre les lui accorde mais le 19 juin le Sénat refuse. Après une première navette, la Chambre confirme son vote mais le Sénat les lui dénie à nouveau. Sans utiliser tous les artifices de procédure pour parvenir à ses fins, Blum annonce sa démission.

35. Archives Syndicat des conserveurs, Quimper, le 9 octobre 1937, le président Schang.

36. Wolikov, Serge, Le Front populaire en France, Paris, Complexe, 1996, p. 260. 
" tente aussi d'imposer sa férule aux populations maritimes. Si elle a assez bien réussi dans le domaine de la marine de commerce, son fiasco est absolu dans celui de la petite pêche ${ }^{37}$ ".

De moins en moins impressionnés, les conserveurs ne s'en laissent plus compter et combattent ouvertement le Syndicat. Les Chambres de Commerce de Brest, Quimper et Lorient s'associent au texte du président de la Chambre de Lyon lorsqu'il dénonce l'ennemi désigné et déclare :

"Permettez-moi de le dire franchement, en dehors de toute préoccupation politique et en parlant au seul nom du bon sens : ou les directives de la CGT sont de la rhétorique et alors elles troublent l'ouvrier et le trompent, ou elles doivent être prises au sérieux et alors elles condamnent d'avance tout réveil de l'esprit d'entreprise ${ }^{38}$."

À Douarnenez, les conserveurs sont parvenus à éviter l'affrontement d'une manière judicieuse en prenant de vitesse la $\mathrm{CGT}^{39}$. Ils ont tout simplement signé, en mai 1937, la convention collective avec la CFTC dont une branche syndicale de l'alimentation est créée cette année-là bien que son implantation ne réponde à aucune réelle demande ouvrière locale. Ils réussissent ainsi à bénéficier d'un texte beaucoup plus favorable à leurs intérêts tout en respectant la loi. Pour autant, les conserveurs ne lâchent rien et, lorsque la CGT revient à la charge, en avril 1938, pour demander des augmentations de salaire, les directeurs décident, " après un échange de vue, de ne pas y répondre ${ }^{40}$ ". Jacques Guillemot, directeur de l'usine quimpéroise Chancerelle, refuse lui aussi de discuter parce que la convention collective pour son établissement a été signée en bonne et due forme et que l'on ne peut toucher aux salaires décidés dans le cadre légal ${ }^{41}$. Son frère, directeur d'une usine à Douarnenez prévient les syndicalistes que

" désormais il se refuserait à discuter avec des gens qui n'exerceraient pas la profession d'une façon effective et constante ${ }^{42}$."

Si la CGT souhaite discuter qu'elle le fasse d'abord avec l'organisation concurrente, la CFTC. Cette opinion est partagée par tous ses collègues du Finistère sud.

" Aucun conflit de travail n'existe à Douarnenez, l'immense majorité du personnel se rendant parfaitement compte de la gravité de la situation; d'ailleurs un contrat collectif, parfaitement régulier, régit leurs rapports avec notre industrie ${ }^{43}$."

37. SERANT, Jacques, La Pêche à la sardine sur les côtes françaises, Quimper, imprimerie cornouaillaise, 1939, p. 59.

38. Archives CCI Brest, discours du président de la Chambre de Lyon lu en séance, septembre 1937.

39. Le Syndicat de l'Alimentation du Finistère, affilié à la CGT n'est créé que le 17 mars 1938.

40. Archives Syndicat des conserveurs, Quimper, le 7 avril 1938, le président Schang.

41. Arch. dép. du Finistère, 10 M 64, Quimper, le 17 août 1938, Jacques Guillemot au secrétaire départemental de la CGT.

42. Archives Syndicat des conserveurs, Quimper, le 27 avril 1938.

43. Arch. dép. du Finistère, 10 M 64, Douarnenez, le 25 août 1938, le Syndicat des fabricants de conserves de Bretagne. 
Marcel Provost, propriétaire d'une usine très importante à Concarneau, renoue avec une ancienne tradition et demande à son syndicat qu'en cas de grèves dans son secteur, il soit possible d'envisager une forme de solidarité entre les conserveurs : ceux qui continuent leurs activités débloquent des fonds pour aider les directeurs des usines fermées. Sa proposition est acquise à l'unanimité sans aucune discussion ${ }^{44}$. Cette proposition n'est ni fortuite ni isolée et participe d'un vaste mouvement national initié par L'Action Patronale, le journal créé en décembre 1936 par Eugène Schueller. Dans le numéro d'avril 1937 on peut y lire le Serment des Patrons, lesquels s'engagent et jurent "d'aider de toutes [leurs] forces et par tous les moyens possibles tout patron en grève ou en lock-out ${ }^{45}$ ". La contre-offensive patronale développée à l'échelon local aussi bien qu'au niveau national relève d'une stratégie mûrie et les conserveurs la respectent à la lettre. Les refus multiples et variés de respecter les accords de Matignon sont autant de motifs pour entraîner des grèves puis des lock-out et enfin les licenciements jugés nécessaires.

Parallèlement les usiniers mettent leurs menaces à exécution, réduisent leur production et n'investissent plus dans la modernisation de leurs chaînes de fabrication. En fait, ils répondent non pas à une quelconque mesure de rétorsion, mais bien à une situation économique peu encourageante. Le poisson en boîte se vend, selon eux, plus mal et les " causes de cette mévente furent imputées, pour des raisons électorales, aux moyens d'absorption insuffisants des usines françaises ${ }^{46}$ ». Les conserveurs n'hésitent pas non plus à augmenter très fortement le prix des boîtes de sardines. Ainsi de Clerville, directeur des conserveries Levesque, une honorable maison nantaise, nous apprend que la boîte de 1/4-25 est vendue au sortir de ses entrepôts 3,30 francs en $1930 ; 3,20$ francs en $1931 ; 2,80$ francs en $1932 ; 2,50$ francs de 1933 à 1935, au plus fort de crise économique pour les usiniers; 3 francs en 1937; 3,60 francs en 1938; 4 francs en $1939^{47}$. La maison Tertrais applique les mêmes taux d'accroissement car elle vend sa boîte du quart 3,13 francs en 1930; 1,98 franc en 1935 et 4,72 francs en $1939^{48}$. La création, le premier juillet 1937, d'un comité central de surveillance des prix confirme cette attitude généralisée de hausse des prix élaborée en 1937 pour compenser les hausses de salaire et largement confirmée dans le monde des conserveurs. D'ailleurs, après de rapides calculs, on s'aperçoit que les hausses décidées par les usiniers entraînent des gains supérieurs aux augmentations accordés aux ouvrières ${ }^{49}$.

44. Archives Syndicat des conserveurs, Quimper, le 10 décembre 1938, le président Schang. 45. L'Action patronale, $\mathrm{n}^{\circ}$ 7, 25 avril 1937 cité dans Kolboom, Ingo, La Revanche des patrons..., op. cit., p. 275.

46. Archives CCI Quimper, séance du 14 mars 1944.

47. M. Babin-Chevaye, gérant de la société de Clerville, Nantes le 13 décembre 1954. Archives fournies par André Marie-d'Avigneau.

48. Patrick Tertrais, Nantes, le 23 décembre 1954. Archives fournies par André Maried'Avigneau.

49. Si on considère que la part de la main d'œuvre nécessaire pour la fabrication d'une boîte vendue 3 francs en 1937 est de trente centimes (soit $10 \%$ du produit final ce qui 
Les mesures sociales et fiscales voulues par le Front populaire font l'objet d'âpres marchandages et les industriels bretons de la conserve apparaissent comme particulièrement intraitables et pugnaces. Ils se contentent de temporiser sans rien céder sur le fond tout en dénonçant les propositions et projets de lois sociales lorsque l'occasion se présente. La presse d'opinion et d' "information " se charge de relayer les diatribes des usiniers ${ }^{50}$, notamment la Dépêche de Brest qui se "fait remarquer par ses insultes envers les grévistes ${ }^{51}$ " et qui réclame l'union de toutes les bonnes volontés pour aider le gouvernement Chautemps :

"Lorsque le cabinet Blum a dû quitter le pouvoir nous étions sous la menace du gouvernement des masses; [...] en moins de trois mois M. Chautemps a écarté cette menace; [...] il a créé une nouvelle atmosphère et $[\ldots]$ aujourd'hui beaucoup de travailleurs [...] commencent à résister aux agitateurs [...]. Il faut lutter contre les colonisateurs communistes ${ }^{52}$."

En octobre 1937, dans un dernier sursaut, le député Lambin ${ }^{53}$ dépose un texte à la Chambre demandant la réduction très nette des droits patronaux. Il est notamment prévu que le patron ne puisse fermer son entreprise sans demander l'avis de la délégation ouvrière, qu'il ne puisse renvoyer un ouvrier sans l'avis de cette même délégation, que l'employeur qui aura fermé son usine ne pourra la rouvrir sans faire appel à son ancien personnel. Des amendes sévères sont prévues pour les patrons récalcitrants. Les Chambres de Commerce de Lorient, de Brest et de Quimper réagissent immédiatement et s'étonnent qu'en cette

" période de pause, il se trouve encore, des démagogues pour battre en brèche l'autorité patronale déjà en agonie... Où cela nous mènera-t-il? À l'emprise par la CGT de tout ce qui concerne l'embauchage et à l'emprise par le même organisme non plus du contrôle mais de la totalité des transactions de tous ordres régissant les entreprises commerciales et industrielles ${ }^{54}$."

\section{L'espoir retrouvé des conserveurs}

Mais la partie est en passe d'être gagnée. La situation politique s'éclaircit encore un peu plus pour les conserveurs lorsque Édouard Daladier est

est un pourcentage supérieur à la réalité), l'augmentation de $15 \%$ des salaires accordée par la maison Levesque représente une plus value de 4,5 centimes par boîte $(0,30$ franc x $15 \%=0,045$ franc). Si l'on rajoute 0,30 franc pour la valeur de l'inflation de $10 \%$ (pourcentage fort) on obtient 34,5 centimes d'augmentation sur le prix final. Et même si l'on tient compte de l'augmentation des prix des boîtes et de l'huile on est encore loin des 50 centimes supplémentaires observés en 1938 !

50. Nogueres, Henri, En France au temps du Front populaire 1935-1938, Paris, Hachette, 1977, p. 251.

51. Arch. dép. du Finistère, 10 M 64, Quimper, le 11 juin 1938, le commissaire au préfet.

52. La Dépêche de Brest et de l'Ouest, le 5 septembre 1937.

53. Cité par les CCI de Quimper, de Brest et de Lorient sans plus de précision sinon que les trois Chambres unanimes demandent le retrait pur et simple de la proposition de loi $\mathrm{n}^{\circ}$ 1045, présentée par Paul Lambin, député SFIO de l'Aisne.

54. Archives CCI Quimper, séance du 5 novembre 1937, le président de Servigny. Archives CCI Brest, séance du 14 décembre 1937, le président Corre. 
nommé président du Conseil le 10 avril 1938. Un climat nouveau se fait sentir dans l'opinion ${ }^{55}$. La " revanche patronale " observée dans bien d'autres secteurs d'activité est également bien engagée par le patronat des conserveries ${ }^{56}$ composé pour l'essentiel d'industriels d'entreprises moyennes. Le point le plus important à régler reste l'augmentation des salaires que les usiniers refusent d'accepter au nom de la survie de leur branche. En mai 1938, l'Union nationale des Fabricants présente un rapport sur la situation comparée de l'exportation des conserves françaises de sardines à l'huile dans lequel tout un paragraphe est consacré à la main d'œuvre :

" Il semble inutile d'indiquer quelle est la charge écrasante qui constitue pour une industrie aussi menacée que la notre, l'incidence conditionnée des diverses charges sociales se superposant au relèvement des bases de salaires; et ceci à tous les stades de la fabrication des conserves ellesmêmes, de leur approvisionnement en matières premières, de leurs conditionnements, de leurs transports et de leur distribution ${ }^{57}$."

On peut d'ailleurs penser qu'un détail anecdotique a joué en faveur des conserveurs; la maîtresse reconnue de Daladier, la marquise de Crusol, est née Jeanne Béziers. Fille, sœur et tante de conserveurs, elle se fit sans doute la fidèle interprète des intérêts des usiniers auprès du président du Conseil ${ }^{58}$. D'autre part, la virulence des ouvriers et ouvrières des conserveries s'amenuise. Ainsi, les 29 octobre et 3 novembre 1938, les décrets d'application de la loi sur les 40 heures dans l'industrie alimentaire sont publiés, il est vrai vidés de leur substance; la situation semble se compliquer pour nos industriels qui, après concertation au sein de la profession et des entretiens rapides avec les administrations concernées, décident de ne rien changer à leur attitude de refus de discussion. On ne touche pas aux salaires, on ne paye pas les heures supplémentaires ou seulement dans des proportions " raisonnables". " Notre position est en fait un droit d'ailleurs parfaitement justifié ${ }^{59}$ " car la branche des conserves alimentaires a obtenu la dérogation tant réclamée pour l'année 1939 : les fameuses heures supplémentaires, si fréquentes dans les conserveries, ne seront pas payées au tarif légal. Pour autant les ouvriers ne réagissent plus et l'annonce des décrets-lois de Paul Reynaud, ministre des Finances, parvient sur le littoral sardinier sans provoquer de réaction notable. Si la loi des

55. MAYeuR, Jean-Marie, La Vie politique sous la Troisième Rébublique, Paris, Le Seuil, 1984, p. 365.

56. BADEL, Laurence, " Grand commerce et pouvoirs publics, 1938-1945 ", dans, MARSELLL, Jacques (dir.), La Révolution commerciale en France, Paris, Le Monde, 1997, p. 144; Kolboom, Ingo, La Revanche des patrons..., op. cit. ; Ruhlmann, Jean, Ni Bourgeois ni prolétaires, Paris, Le Seuil, 2001.

57. Archives FIAC (Fédération française des Industries d'Aliments Conservés), rapport du 5 mai 1938. Et d'ailleurs les conserveurs se contentent de rappeler le fait mais ils ne précisent jamais cette fameuse part de la main d'œuvre dans le prix du produit fini car en fait elle est très faible, de 6 à $9 \%$ en 1936 !

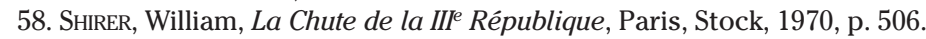

59. Archives CCI Quimper, le 17 novembre 1938, le président De Servigny, conserveur à Concarneau. 
40 heures n'est pas abrogée, elle perd toute signification car les assouplissements et les dérogations prévues permettent de la contourner sinon de l'ignorer. La tarification des heures supplémentaires, cheval de bataille des fabricants de sardines à l'huile qui emploient une main d'œuvre saisonnière pendant de longues journées de travail l'été, est elle aussi fortement diminuée.

Pourtant, tous les conserveurs savent pertinemment que les discussions avec la CGT sont inévitables car les accords signés avec la CFTC prennent fin en mars 1938, et le nouvel interlocuteur pour la formation des commissions arbitrales et la renégociation des conventions collectives sera forcément le syndicat honni. Dès la fin de l'année 1938, les tensions sont tellement exacerbées que les fabricants ne peuvent plus éviter la rencontre mais, avant d'être placés devant le fait accompli, ils se voilent la face sans se préoccuper de l'avenir social des ouvriers. Les faits semblent leur donner raison. La grève générale du 30 novembre 1938, organisée par la CGT pour répondre aux décrets-lois Reynaud du 12 novembre 1938, est un échec particulièrement cuisant dans le monde ouvrier sardinier; on ne constate aucun mouvement de soutien, aucune occupation d'usine, aucune manifestation d'aucune sorte ${ }^{60}$. Le désarroi des travailleurs est extrême face à l'unanimité des vues d'un patronat français retrouvé et confiant ${ }^{61}$. C'était pour lui dorénavant que " tout était possible ${ }^{62}$ ". De fait, la résistance et la collaboration patronales fonctionnent très bien dès qu'il s'agit de défendre " les intérêts du commerce, de l'industrie et du travail ». Les conserveurs affirment avec force et sans état d'âme leurs convictions : Charles de Servigny, conserveur à Concarneau et président de la Chambre de commerce de Quimper se félicite de l'issue de la bataille patronale menée depuis deux ans par ses collègues et lui-même : "C'est avec un réel plaisir que j'ai constaté une opposition très nette aux propositions du gouvernement [Blum] ${ }^{63}$. " On se contente dès lors d'enfoncer le clou et de vitupérer les syndicalistes en constatant

" les efforts toujours faits en sus par tous ceux qui luttent pour le soi-disant bonheur de la classe ouvrière. Il faut aussi signaler la manière hypocrite de préparer les esprits en s'infiltrant de façon insidieuse chaque année ${ }^{64}$ ".

Si l'on se réfère aux statistiques de l'Union des Syndicats français de Fabricants de Conserves de Poissons on constate d'ailleurs que les années du Front populaire sont loin d'être mauvaises pour les industriels; il existe 142 usines ouvertes en 1935 mais on en compte 151 en 1938. La production

60. Arch. dép. du Finistère, 10 M 71, grève générale du 30 novembre 1938.

61. Au niveau national on constate d'ailleurs la même abstention; seulement $9,9 \%$ des syndiqués CGT de la branche de l'Alimentation suivent cette grève nationale. Cité dans KERGOAT, Jacques, La France du Front populaire, op. cit., p. 286.

62. Selon le mot de Marceau Pivert, dans le Populaire du 27 mai 1936.

63. Archives CCI Quimper, le 13 décembre 1938, le président de Servigny.

64. Archives Syndicat des conserveurs, Quimper, le 10 décembre 1938, le président Schang. 
ne cesse de s'accroître au cours de la période malgré les cris d'alarme de la profession ${ }^{65}$. Les exportations de sardines à l'huile connaissent le même mouvement, passant de 1280 tonnes en 1936 à 1760 tonnes en 1937 et 1850 tonnes en $1938^{66}$. Il faut donc croire que cette attitude du gros dos et du refus systématique présente certains avantages puisque les affaires restent florissantes et que les accords définitifs ne sont toujours pas signés entre les conserveurs et la CGT en 1939. Après un premier moment de fébrilité, les fabricants se sont repris très vite et ont appliqué à la lettre les directives de leurs syndicats régionaux et de l'Union nationale qui se sont montrés d'une redoutable efficacité, deux mois après l'installation du gouvernement Blum, pour le combattre sans ménagement. Ces organisations, déjà anciennes ${ }^{67}$, sont capables, dès juillet 1936 , d'élaborer " une architecture professionnelle " que les difficultés du Front populaire n'ont fait que confirmer ${ }^{68}$. Une ligne dure, sans concession aucune sauf nécessité absolue, est définie dès l'été 1936 et les conserveurs, syndiqués à plus de $90 \%{ }^{69}$, s'y tiennent. Cette attitude résolue et unanime est exemplaire et mérite d'être signalée dans une profession composée de petites et moyennes entreprises. La fermeté auprès d'une population ouvrière peu organisée et disséminée sur tout le littoral de la Bretagne méridionale s'avère très " adaptée ", en termes patronaux. Le retour à une production normale s'effectue sans que les usiniers n'aient eu beaucoup à céder sur les revendications salariales; leurs prérogatives sont encore pratiquement intactes.

Les bruits de bottes allemandes à nos frontières repoussent la résolution des problèmes sociaux à des jours meilleurs. Dorénavant, les pouvoirs publics entendent organiser la Nation en temps de guerre et plus particulièrement sur le terrain de la production industrielle pour ce qui nous intéresse. L'effort demandé à toute la population ne peut souffrir de contestations : pour la Victoire, sans récrimination, la France doit travailler unie, dont les ouvrières des conserveries, mais pour quels salaires.

65. Archives FIAC. Union des Syndicats français de fabricants de Conserves de Poissons, Rapport d'activité pour l'année 1938, Paris, février 1939.

66. Tableaux du Commerce de la France, ministère du Commerce.

67. Le Syndicat des fabricants de sardines à l'huile est créé le premier à Nantes dès 1875; l'année suivante est fondé celui des fabricants de la région parisienne, puis en 1888 apparaissent ceux des conserveurs bretons et bordelais.

68. Archives CCI Quimper. Paris, le 13 mai 1939, lettre de Louis de Clerville, président de l'Union des syndicats français des fabricants de conserves de sardines et autres poissons.

69. Archives FIAC. En 1936, 149 usines ouvertes appartenant à des membres syndiqués et 9 usines hors syndicats. 


\section{RESUME}

L'industrie des conserves de sardines à l'huile est née en France en 1824, à Nantes, dans la friterie de Pierre-Joseph Colin. Très rapidement cette branche d'activité connaît un développement considérable et la production monte en flêche pour dépasser les 20000 tonnes en 1900 à l'apogée de la fabrication française. En 1936 les 150 usines installées sur le littoral atlantique fabriquent encore 12000 tonnes de sardines à l'huile et les dirigeants de ces conserveries forment un patronat soudé qui se retrouve uni et opposé aux réformes menées par le Front populaire. Bien sûr leur nombre est peu important, une centaine, mais cette faiblesse numérique est aussi un avantage car elle permet de suivre clairement leurs actes, emblématiques de toute la classe dirigeante industrielle. Cet aspect peu connu de la révolte d'un groupe particulier de patrons nous renseigne plus précisément sur leur attitude d'opposition systématique, engagée dès juillet 1936, et nous explique, à une échelle réduite, les mécanismes qui ont annihilé les effets des grandes lois sociales votées par le Front populaire.

\section{ABSTRACT}

The sardine canning industry started out in France in 1824, in Nantes, in P. J. Colin's friying factory. Very soon, this branch of activity expanded considerably and the production shot up and exceeded 20.000 tons in 1900, when french production reached its peak. In 1936, the 150 factories located on the Atlantic coastline were still producing 12.000 tons of sardines in oil and the directors of those canning factories formed a strongly united group that opposed the reforms carried out by the Popular Front. Of course, they were not very numerous, about a hundred, but at the same time, their small number is an adventage as it allows to follow clearly their acts, represantative of the industrial ruling class as a whole. This littleknown aspect of the revolt of a particular group of employers provides us with detailed informations about their attitude of systematic opposition, adopted as early as July 1936, and it clarifies, on a small scale, the mechanisms which ruined the effects of the main social laws voted by the Popular Front. 
